\title{
ON EXISTENCE OF EXTREMAL SOLUTIONS OF NONLINEAR FUNCTIONAL INTEGRAL EQUATIONS IN BANACH ALGEBRAS
}

\author{
B. C. DHAGE
}

Received 23 August 2003 and in revised form 9 May 2004

An algebraic fixed point theorem involving the three operators in a Banach algebra is proved using the properties of cones and they are further applied to a certain nonlinear integral equations of mixed type $x(t)=k(t, x(\mu(t)))+[f(t, x(\theta(t)))]\left(q(t)+\int_{0}^{\sigma(t)} v(t\right.$, $s) g(s, x(\eta(s))) d s)$ for proving the existence of maximal and minimal solutions. Our results include the earlier fixed point theorems of Dhage (1992 and 1999) as special cases with a different but simple method.

\section{Introduction}

It is known that the algebraic methods are useful for proving the existence of extremal solutions for various classes of differential and integral equations under certain monotonicity conditions. The exhaustive account of this subject appears in Amann [1], Deimling [4], and Heikkilä and Lakshmikantham [12] and the references therein. The existence theorems for nonlinear integral equations of mixed type are generally obtained by using the fixed point theorems of Krasnosel'skii [13] and Dhage [6, 8]. Most recently, the author in $[6,7]$ proved some fixed point theorems involving two operators in a Banach algebra via the use of measure of noncompactness and they are further applied to a certain nonlinear integral equation in Banach algebras for proving the existence of extremal solutions under the mixed algebraic and topological conditions, such as monotonicity and continuity of the nonlinearities involved in the equation. In this paper, we generalize the fixed point theorem of Dhage $[6,7]$ to three operators under the weaker conditions with a different method and apply the newly developed fixed point theorem to a certain nonlinear integral equation in Banach algebras for proving the existence of extremal solutions.

\section{Abstract results}

Let $X$ be a Banach space with norm $\|\cdot\|$. A nonempty closed subset $K$ of Banach algebra $X$ is called a cone if 
272 Extremal solutions of integral equations

(i) $K+K \subseteq K$,

(ii) $\lambda K \subseteq K$ whenever $\lambda \in \mathbb{R}$ and $\lambda \geq 0$,

(iii) $-K \cap K=\{0\}$, where 0 is a zero element of $X$.

Further, a cone $K$ is called positive if

(iv) $K \circ K \subset K$, where $\circ$ is the multiplicative composition in $X$.

We define an order relation $\leq$ in $X$ as follows. Let $x, y \in X$. Then

$$
x \leq y \Longleftrightarrow y-x \in K
$$

Notice that condition (iv) implies that if $x \leq y$ and $z \in K$, then $x z \leq y z$.

A cone $K$ is normal if the norm in $X$ is semimonotone on $K$, that is, if $0 \leq x \leq y$, then $\|x\| \leq N\|y\|$ for some real number $N>0$. The details of cones and their properties may be found in the monographs like Guo and Lakshmikantham [11] and Heikkilä and Lakshmikantham [12].

Let $u, v \in X$ be such that $u \leq v$. Then the set

$$
[u, v]=\{x \in X \mid u \leq x \leq v\}
$$

is called an order interval in $X$. Since $K$ is closed, every order interval is closed in $X$.

Definition 2.1. A mapping $T: X \rightarrow X$ is called increasing if for all $x, y \in X$, Tx $\leq$ Ty whenever $x \leq y$.

A mapping $A: X \rightarrow X$ is called $\mathscr{D}$-Lipschitzian if there exists a continuous nondecreasing function $\phi: \mathbb{R}^{+} \rightarrow \mathbb{R}^{+}$satisfying

$$
\|A x-A y\| \leq \phi_{A}(\|x-y\|)
$$

for all $x, y \in X$ with $\phi_{A}(0)=0$. Sometimes the function $\phi_{A}$ is called a $\mathscr{D}$-function of $A$ on $X$. In the special case when $\phi_{A}(r)=\alpha r, \alpha>0, A$ is called a Lipschitzian with a Lipschitz constant $\alpha$. In particular, if $\alpha<1, A$ is called a contraction with a contraction constant $\alpha$. Further, if $\phi_{A}(r)<r$ for $r>0$, then $A$ is called a nonlinear contraction on $X$.

The following fixed point theorem for the nonlinear contraction is well known and is useful for proving the existence and the uniqueness theorems for the nonlinear differential and integral equations.

Theorem 2.2 (Boyd and Wong [2]). Let $A: X \rightarrow X$ be a nonlinear contraction. Then $A$ has a unique fixed point $x^{*}$ and the sequence $\left\{A^{n} x\right\}$ of successive iterations of $A$ converges to $x^{*}$ for each $x \in X$.

An operator $T: X \rightarrow X$ is called compact if $\overline{T(X)}$ is a compact subset of $X$. Similarly, $T: X \rightarrow X$ is called totally bounded if $T$ maps a bounded subset of $X$ into the relatively compact subset of $X$. Finally, $T: X \rightarrow X$ is called completely continuous operator if it is continuous and totally bounded operator on $X$. However, the two notions of compactness and total boundedness of $T$ are equivalent on bounded subsets of $X$.

The following theorem of [7] is well known and is useful in the theory of nonlinear differential and integral equations in Banach algebras. 
We note that an operator $T$ on a Banach space $X$ into itself is called positive if $\operatorname{range}(T) \subseteq K$.

Theorem 2.3. Let the order cone $K$ of a real algebra $X$ be positive and normal, and let $u, v \in X$ be such that $u \leq v$. Let $A, B:[u, v] \rightarrow X$ be two positive operators such that

(a) $A$ is $\mathscr{D}$-Lipschitzian and increasing,

(b) $B$ is completely continuous and increasing,

(c) $M \phi_{A}(r)<r$ for $r>0$, where $M=\|B([u, v])\|=\sup \{\|B(x)\|: x \in[u, v]\}$,

(d) $A x B x \in[u, v]$ for all $x \in[u, v]$.

Then operator equation $A x B x=x$ has a least solution and a greatest one in $[u, v]$.

THeOREM 2.4. Let the order cone $K$ of a real algebra $X$ be positive and normal, and let $u, v \in X$ be such that $u \leq v$. Let $A, B:[u, v] \rightarrow K$ and $C:[u, v] \rightarrow X$ be three operators such that

(a) $A$ is D-Lipschitzian and increasing,

(b) $B$ is completely continuous and increasing,

(c) $C$ is D-Lipschitzian and increasing,

(d) $M \phi_{A}(r)+\phi_{C}(r)<r$ for $r>0$, where $M=\|B([u, v])\|=\sup \{\|B(x)\|: x \in[u, v]\}$,

(e) $u \leq A u B u+C u$ and $A v B v+C v \leq v$.

Then operator equation $A x B x+C x=x$ has a least solution and a greatest one in $[u, v]$.

Proof. Clearly $[u, v]$ is convex and closed subset of $X$ which is further bounded in view of the normality of the cone $K$ in $X$.

Let $y \in[u, v]$ be a fixed element. Define an operator $A_{y}:[u, v] \rightarrow X$ by

$$
A_{y}(x)=A x B y+C x, \quad x \in[u, v] .
$$

Since $A$ and $B$ are positive and increasing, one has

$$
A_{y}(u)=A x B y+C x \leq A v B y+C v=A_{y}(v) .
$$

Moreover, condition (e) implies that

$$
u \leq A u B u+C u \leq A u B y+C u \leq A v B v+C v \leq v .
$$

Hence $A_{y}$ defines a mapping $A_{y}:[u, v] \rightarrow[u, v]$. Then for any $x_{1}, x_{2} \in[u, v]$, we have

$$
\begin{aligned}
\left\|A_{y}\left(x_{1}\right)-A_{y}\left(x_{2}\right)\right\| & =\left\|A x_{1} B y-A x_{2} B y\right\|+\left\|C x_{1}-C x_{2}\right\| \\
& \leq\left\|A x_{1}-A x_{2}\right\|\|B y\|+\left\|C x_{1}-C x_{2}\right\| \\
& \leq M \phi_{A}\left(\left\|x_{1}-x_{2}\right\|\right)+\phi_{C}\left(\left\|x_{1}-x_{2}\right\|\right) .
\end{aligned}
$$

This shows that $A_{y}$ is a nonlinear contraction on $X$ in view of the hypothesis (c). Therefore, an application of Theorem 2.2 yields that $A_{y}$ has a unique fixed point, say $x^{*}$, in $[u, v]$ and the sequence $\left\{A_{y}^{n}(u)\right\}$ converges to $x^{*}$. By the definition of $A_{y}$,

$$
A_{y}\left(x^{*}\right)=A x^{*} B y+C x^{*}=x^{*} .
$$


Similarly, if $w \in[u, v]$, then the operator $A_{w}(x)=A x B w+C x$ is a nonlinear contraction on $[u, v]$ into itself and the sequence $\left\{A_{w}^{n}(u)\right\}$ converges to a unique fixed point $z^{*}$ of $A_{w}$. If $y \leq w$, then

$$
A_{y}(u)=A u B y+C u \leq A u B w+C u=A_{w}(u) .
$$

It is easy to prove that $A_{y}^{n}(u) \leq A_{w}^{n}(u)$ for all $n \in \mathbb{N}$. Hence we have

$$
x^{*}=\lim _{n \rightarrow \infty} A_{y}^{n}(u) \leq \lim _{n \rightarrow \infty} A_{w}^{n}(u)=z^{*} .
$$

Define a mapping $N:[u, v] \rightarrow X$ by $N y=z$, where $z$ is the unique solution of the equation $z=A z B y+C z, z \in[u, v]$. We show that $N$ is continuous on $[u, v]$. Let $\left\{y_{n}\right\}$ be a sequence in $[u, v]$ converging to a point $y$.

Now

$$
\begin{aligned}
\left\|N y_{n}-N y\right\|= & \left\|A N\left(y_{n}\right) B y_{n}-A N(y) B y\right\|+\left\|C\left(N y_{n}\right)-C(N y)\right\| \\
\leq & \left\|A N\left(y_{n}\right) B y_{n}-A N(y) B y_{n}\right\|+\left\|A N(y) B y_{n}-A N(y) B y\right\| \\
& +\left\|C\left(N y_{n}\right)-C(N y)\right\| \\
\leq & \left\|A N y_{n}-A N(y)\right\|\left\|B y_{n}\right\|+\|A N(y)\|\left\|B y_{n}-B y\right\| \\
& +\left\|C\left(N y_{n}\right)-C(N y)\right\| \\
\leq & M \phi_{A}\left(\left\|N y_{n}-N y\right\|\right)+\|A N y\|\left\|B y_{n}-B y\right\| \\
& +\phi_{C}\left(\left\|N y_{n}-N y\right\|\right) .
\end{aligned}
$$

Taking the limit superior as $n \rightarrow \infty$ and using the fact that $\phi_{A}$ and $\phi_{C}$ are continuous functions, we obtain

$$
\begin{aligned}
\underset{n \rightarrow \infty}{\limsup }\left\|N y_{n}-N y\right\| \leq & M \phi_{A}\left(\limsup _{n \rightarrow \infty}\left\|N y_{n}-N y\right\|\right) \\
& +\|A N y\|\left(\limsup _{n \rightarrow \infty}\left\|B y_{n}-B y\right\|\right) \\
& +\phi_{C}\left(\limsup _{n \rightarrow \infty}\left\|N y_{n}-N y\right\|\right) .
\end{aligned}
$$

Now from hypothesis (c), it follows that $\lim _{n \rightarrow \infty}\left\|N y_{n}-N y\right\|=0$. This shows that $N$ is continuous on $[u, v]$. Next we show that $N$ is totally bounded on $[u, v]$. Let $S$ be any subset of $[u, v]$. Since $[u, v]$ is norm bounded, $S$ is a norm bounded subset of $[u, v]$. Now for any $z \in S$, we have

$$
\|A z\| \leq\|A a\|+\|A z-A a\| \leq\|A a\|+\alpha\|z-a\| \leq c,
$$

where $c=\|A a\|+\alpha \operatorname{diam}(S)$ and $a \in S$, a fixed element.

Let $\epsilon>0$ be given. Since $B$ is completely continuous, $B(S)$ is totally bounded. Then there is a set $Y=\left\{y_{1}, \ldots, y_{n}\right\}$ in $S$ such that

$$
B(S) \subset \bigcup_{i=1}^{n} \mathscr{\Re}_{\delta}\left(w_{i}\right),
$$


where $w_{i}=B\left(y_{i}\right)$ and $\delta=((1-(\alpha M+\beta)) / c) \epsilon$ and $\mathscr{B}_{\delta}\left(w_{i}\right)$ is an open ball centered at $w_{i}$ of radius $\delta$. Therefore, for any $y \in S$, we have a $y_{k} \in Y$ such that

$$
\left\|B y-B y_{k}\right\| \leq\left(\frac{1-(\alpha M+\beta)}{c}\right) \epsilon
$$

Also we have

$$
\begin{aligned}
\left\|N y-N y_{k}\right\| & \leq\left\|A z B y-A z_{k} B y_{k}\right\|+\left\|C z-c z_{k}\right\| \\
& \leq\left\|A z B y-A z_{k} B y\right\|+\left\|A z_{k} B y-A z_{k} B z_{k}\right\|+\left\|C z-c z_{k}\right\| \\
& \leq\left\|A z-A z_{k}\right\|\|B y\|+\left\|A z_{k}\right\|\left\|B y_{k}-B y\right\|+\left\|C z_{k}-c z\right\| \\
& \leq(\alpha M+\beta)\left\|z-z_{k}\right\|+\|A z\|\left\|B y_{k}-B y\right\| \\
& \leq \frac{c}{1-(\alpha M+\beta)}\left\|B y-B y_{k}\right\|<\epsilon .
\end{aligned}
$$

This is true for every $y \in S$ and hence

$$
N(S) \subset \bigcup_{i=1}^{n} \mathscr{B}\left(z_{i}\right),
$$

where $z_{i}=N y_{i}$. As a result, $N(S)$ is totally bounded. Since $N$ is continuous, it is a compact operator on $S$. Moreover, $N$ is increasing because $A, B$, and $C$ are increasing on $[u, v]$. Hence $\left\{N^{n}(u)\right\}$ is an increasing sequence and $\left\{N^{n}(v)\right\}$ is a decreasing sequence in $N([u, v]) \subset[u, v]$. Now the limits of these sequences exist (see Heikkilä and Lakshmikantham [12]) and they belong to the order interval $[u, v]$.

Denote

$$
x_{*}=\lim _{n \rightarrow \infty} N^{n} u, \quad x^{*}=\lim _{n \rightarrow \infty} N^{n} v .
$$

By the continuity of $N$, we obtain

$$
\begin{aligned}
& N x_{*}=N\left(\lim _{n \rightarrow \infty} N^{n} u\right)=\lim _{n \rightarrow \infty} N^{n+1}(u)=x_{*}, \\
& N x^{*}=N\left(\lim _{n \rightarrow \infty} N^{n} v\right)=\lim _{n \rightarrow \infty} N^{n+1}(v)=x^{*},
\end{aligned}
$$

and since $N$ is increasing, it follows that $x_{*} \leq x^{*}$. Assume that $x \in[u, v]$ is a fixed point of the operator $N$. Then by the definition of $S$,

$$
x=N x=A(N x) B x+C(N x)=A x B x+C x,
$$

and so the operator equation $x=A x B x+C x$ has a solution in $[u, v]$. As a result, $x_{*}$ and $x^{*}$ are solutions of the operator equation $A x B x+C x=x$.

Assume that $z \in[u, v]$ is a solution of the operator equation $A x B x+C x=x$. Then from the definitions of $N$, it follows that $z$ is also a fixed point of $N$. Since $u \leq z \leq v$, we have $N u \leq N z=z \leq N v$. Proceeding in this way, by induction, we get $N^{n} u \leq z \leq N^{n} v$, and so $x_{*} \leq x^{*}$. Thus $x_{*}$ is the least solution and $x^{*}$ is the greatest one of the equation $A x B x+C x=x$ in $[u, v]$. This completes the proof. 
Notice that the proof of Theorem 2.3 involves only the elementary ideas of functional analysis and does not involve the use of the advanced notion of measure of noncompactness in Banach spaces.

As a consequence of Theorem 2.3, we obtain Corollary 2.5 in its applicable form to the nonlinear equations.

Corollary 2.5. Let the order cone $K$ of a real algebra $X$ be positive and normal, and let $u, v \in X$ be such that $u \leq v$. Let $A, B: X \rightarrow K$ and $C: X \rightarrow X$ be three increasing operators such that

(a) A and $B$ are Lipschitzians with Lipschitz constants $\alpha$ and $\beta$, respectively,

(b) $B$ is completely continuous,

(c) $\alpha M+\beta<1$, where $M=\|B([u, v])\|=\sup \{\|B(x)\|: x \in[u, v]\}$,

(e) $u \leq A u B u+C u$ and $A v B v+C v \leq v$.

Then operator equation $A x B x+C x=x$ has a least solution and a greatest one in $[u, v]$.

When $C \equiv 0$ and $\phi(r)=\alpha r, 0 \leq \alpha<1$, in Theorem 2.4, we get the interesting Corollary 2.6 to [7, Theorem 2.3] which has numerous applications in the theory of nonlinear differential and integral equations.

Corollary 2.6. Let the order cone $K$ of a real Banach algebra $X$ be positive and normal, and let $u, v \in X$ be such that $u \leq v$. Let $A, B: X \rightarrow K$ be two increasing operators such that

(a) $A$ is Lipschitzian with a Lipschitz constant $\alpha$ and is increasing on $[u, v]$,

(b) $B$ is continuous and compact on $[u, v]$,

(c) $\alpha M<1$, where $M=\|B([u, v])\|=\sup \{\|B(x)\|: x \in[u, v]\}$,

(d) $u \leq A u B u$ and $A v B v \leq v$.

Then operator equation $A x B x=x$ has a least solution and a greatest one in $[u, v]$.

\section{Functional integral equations}

Let $\mathbb{R}$ denote the real line. Given a closed and bounded interval $J=[0,1]$ in $\mathbb{R}$, consider the nonlinear functional integral equation (in short FIE)

$$
x(t)=k(t, x(\mu(t)))+[f(t, x(\theta(t)))]\left(q(t)+\int_{0}^{\sigma(t)} v(t, s) g(s, x(\eta(s))) d s\right)
$$

for all $t \in J$, where $\mu, \theta, \sigma, \eta: J \rightarrow J, q: J \rightarrow \mathbb{R}, v: J \times J \rightarrow \mathbb{R}$, and $f, g, k: J \times \mathbb{R} \rightarrow \mathbb{R}$.

The special cases of the FIE (3.1) occur in some natural, physical, and social sciences; see Chandrasekhar [3] and Deimling [4] and the references therein. The FIE (3.1) and some of its special cases have been discussed in Dhage $[5,8]$ and Dhage and O'Regan [9] for the existence results. In this section, we will prove the existence theorem for the extremal solutions of FIE (3.1) by an application of the abstract fixed point theorem embodied in Corollary 2.5.

Let $M(J, \mathbb{R})$ and $B(J, \mathbb{R})$ denote, respectively, the spaces of all measurable and bounded real-valued functions on $J$. We will seek the solution of FIE $(3.1)$ in the space $B M(J, \mathbb{R})$ of 
bounded and measurable real-valued functions on $J$. Define a norm

$$
\|x\|_{B M}=\max _{t \in J}|x(t)| .
$$

Clearly, $B M(J, \mathbb{R})$ is a Banach algebra with this maximum norm. We define an order relation $\leq$ in $B M(J, \mathbb{R})$ with the help of the cone $K$ in $B M(J, \mathbb{R})$ defined by

$$
K=\{x \in B M(J, \mathbb{R}) \mid x(t) \geq 0 \forall t \in J\}
$$

Clearly, $K$ is a positive and normal cone in $B M(J, \mathbb{R})$. Let $L(J, \mathbb{R})$ denote the space of Lebesgue integrable real-valued functions on $J$, with a norm $\|\cdot\|_{L^{1}}$ defined by

$$
\|x\|=\int_{0}^{1}|x(t)| d t
$$

We need the following definitions in the sequel.

Definition 3.1. A mapping $\beta: J \times \mathbb{R} \rightarrow \mathbb{R}$ is said to satisfy a condition of Carathéodory or simply is called Carathéodory if

(i) $t \mapsto \beta(t, x)$ is measurable for each $x \in \mathbb{R}$,

(ii) $x \mapsto \beta(t, x)$ is continuous almost everywhere for $t \in J$.

Further, a Carathéodory function $\beta(t, x)$ is called $L^{1}$-Carathéodory if

(iii) for each real number $r>0$, there exists a function $h_{r} \in L^{1}(J, \mathbb{R})$ such that

$$
|\beta(t, x)| \leq h_{r}(t) \quad \text { a.e. } t \in J
$$

for all $x \in \mathbb{R}$ with $|x| \leq r$.

Definition 3.2. A solution $x_{M}$ of FIE (3.1) is called maximal if $x$ is any other solution; then $x(t) \leq x_{M}(t)$ for all $t \in J$. Similarly, a solution $x_{m}$ of FIE (3.1) is called minimal if $x$ is any other solution; then $x_{m}(t) \leq x(t)$ for all $t \in J$.

Definition 3.3. A function $a \in B M(J, \mathbb{R})$ is called a lower solution of FIE (3.1) if

$$
a(t) \leq k(t, a(\mu(t)))+[f(t, a(\theta(t)))]\left(q(t)+\int_{0}^{\sigma(t)} v(t, s) g(s, a(\eta(s))) d s\right)
$$

for all $t \in J$. Similarly, a function $b \in B M(J, \mathbb{R})$ is called an upper solution of FIE (3.1) if

$$
b(t) \geq k(t, b(\mu(t)))+[f(t, b(\theta(t)))]\left(q(t)+\int_{0}^{\sigma(t)} v(t, s) g(s, b(\eta(s))) d s\right)
$$

for all $t \in J$.

We consider the following set of assumptions.

$\left(\mathrm{H}_{0}\right)$ The functions $\mu, \theta, \sigma, \eta: J \rightarrow J$ are continuous.

$\left(\mathrm{H}_{1}\right)$ The function $q: J \rightarrow \mathbb{R}^{+}$is continuous with $Q=\sup _{t \in J}|q(t)|$.

$\left(\mathrm{H}_{2}\right)$ The function $v: J \times J \rightarrow \mathbb{R}^{+}$is continuous and $V=\sup _{t, s \in J}|v(t, s)|$. 
$\left(\mathrm{H}_{3}\right)$ The function $k: J \times \mathbb{R} \rightarrow \mathbb{R}^{+}$is continuous and there is a function $\beta_{1} \in B(J, \mathbb{R})$ with bound $\left\|\beta_{1}\right\|$ such that

$$
|k(t, x)-k(t, y)| \leq \beta_{1}(t)|x-y| \quad \text { a.e. } t \in J
$$

for all $x, y \in \mathbb{R}$.

$\left(\mathrm{H}_{4}\right)$ The function $f: J \times \mathbb{R} \rightarrow \mathbb{R}^{+}-\{0\}$ is continuous and there is a function $\alpha_{1} \in$ $B(J, \mathbb{R})$ with bound $\left\|\alpha_{1}\right\|$ such that

$$
|f(t, x)-f(t, y)| \leq \alpha_{1}(t)|x-y| \quad \text { a.e. } t \in J
$$

for all $x, y \in \mathbb{R}$.

$\left(\mathrm{H}_{5}\right)$ The function $g: J \times \mathbb{R} \rightarrow \mathbb{R}^{+}$is Carathéodory.

$\left(\mathrm{H}_{6}\right)$ The functions $f(t, x), g(t, x)$, and $k(t, x)$ are nondecreasing in $x$ almost everywhere for $t \in J$.

$\left(\mathrm{H}_{7}\right)$ FIE (3.1) has a lower solution $u$ and an upper solution $v$ with $u \leq v$.

Remark 3.4. Suppose that hypotheses $\left(\mathrm{H}_{5}\right),\left(\mathrm{H}_{6}\right)$, and $\left(\mathrm{H}_{7}\right)$ hold. Then the function $h$ : $J \rightarrow \mathbb{R}$, defined by

$$
h(t)=|g(t, a(t))|+|g(t, b(t))| \quad \text { a.e. } t \in J,
$$

is Lebesgue integrable and

$$
|g(t, x)| \leq h(t)
$$

for all $t \in J$ and for all $x \in[a, b]$.

Theorem 3.5. Assume that the hypotheses $\left(\mathrm{H}_{0}\right)-\left(\mathrm{H}_{7}\right)$ hold. If

$$
\left\|\alpha_{1}\right\|\left(Q+V\|h\|_{L^{1}}\right)+\left\|\beta_{1}\right\|<1
$$

then FIE (3.1) has a minimal solution and a maximal one in $[a, b]$.

Proof. Consider the order interval $[a, b]$ in $B M(J, \mathbb{R})$ which is well defined in view of hypothesis $\left(\mathrm{H}_{7}\right)$. Define three operators $A, B$, and $C$ on $B M(J, \mathbb{R})$ by

$$
\begin{aligned}
& A x(t)=f(t, x(\theta(t))), \quad t \in J, \\
& B x(t)=q(t)+\int_{0}^{\sigma(t)} v(t, s) g(s, x(\eta(s))) d s, \quad t \in J, \\
& C x(t)=k(t, x(\mu(t))), \quad t \in J .
\end{aligned}
$$

Then the FIE (3.1) is equivalent to the operator equation

$$
A x(t) B x(t)+C x(t)=x(t), \quad t \in J .
$$


We will show that the operators $A, B$, and $C$ satisfy all the conditions of Theorem 2.4 on $B M(J, \mathbb{R})$. Since the functions $q, v, f$, and $g$ are nonnegative, $A$ and $B$ define the operators $A, B: B M(J, \mathbb{R}) \rightarrow K$. Let $x, y \in X$ be such that $x \leq y$. Then we have

$$
A x(t)=f(t, x(\theta(t))) \leq f(t, y(\theta(t)))=B y(t)
$$

for all $t \in J$. Hence $A$ is increasing on $X$. Similarly, it is shown that the operators $B$ and $C$ are also increasing on $X$. Let $x, y \in B M(J, \mathbb{R})$. Then by $\left(\mathrm{H}_{4}\right)$,

$$
\begin{aligned}
|A x(t)-A y(t)| & =|f(t, x(\theta(t)))-f(t, y(\theta(t)))| \\
& \leq \alpha_{1}(t)|x(\theta(t))-y(\theta(t))| \\
& \leq\left\|\alpha_{1}||\right\| x-y \|_{B M .}
\end{aligned}
$$

Taking the maximum over $t$,

$$
\|A x-A y\|_{B M} \leq\left\|\alpha_{1}\right\|\|x-y\|_{B M} .
$$

This shows that $A$ is a Lipschitzian with a Lipschitz constant $\left\|\alpha_{1}\right\|$. Similarly, it is shown that $C$ is a Lipschitzian with a Lipschitz constant $\left\|\beta_{1}\right\|$.

Next we will show that the operator $B$ is continuous and compact on $[u, v]$. Since $g(t, x)$ is $L^{1}$-Carathéodory, by using the dominated convergence theorem (see Granas et al. $[10])$, it can be shown that $B$ is continuous on $B M(J, \mathbb{R})$. Let $\left\{x_{n}\right\}$ be a sequence in $[u, v]$. Then by Remark 3.4,

$$
\begin{aligned}
\left|B x_{n}(t)\right| & \leq|q(t)|+\left|\int_{0}^{\sigma(t)}\right| v(t, s)|| g(s, x(\eta(s)))|d s| \\
& \leq Q+\int_{0}^{\sigma(t)} h(s) d s \leq Q+V\|h\|_{L^{1}}
\end{aligned}
$$

which further yields that $\left\|B x_{n}\right\| \leq Q+V\|h\|_{L^{1}}$ for each $n \in \mathbb{N}$. As a result, $\left\{B x_{n}: n \in \mathbb{N}\right\}$ is a uniformly bounded set in $B M(J, \mathbb{R})$. Let $t, \tau \in J$. Then by the definition of $B$,

$$
\begin{aligned}
\left|B x_{n}(t)-B x_{n}(\tau)\right| \leq & |q(t)-q(\tau)| \\
& +\left|\int_{0}^{\sigma(t)} v(t, s) g(s, x(\eta(s))) d s-\int_{0}^{\sigma(\tau)} v(t, s) g(s, x(\eta(s))) d s\right| \\
\leq & |q(t)-q(\tau)| \\
& +\left|\int_{0}^{\sigma(t)} v(t, s) g(s, x(\eta(s))) d s-\int_{0}^{\sigma(t)} v(\tau, s) g(s, x(\eta(s))) d s\right| \\
& +\left|\int_{0}^{\sigma(t)} v(\tau, s) g(s, x(\eta(s))) d s-\int_{0}^{\sigma(\tau)} v(\tau, s) g(s, x(\eta(s))) d s\right|
\end{aligned}
$$




$$
\begin{aligned}
& \leq|q(t)-q(\tau)|+\int_{0}^{\sigma(t)}|v(t, s)-v(\tau, s)||g(s, x(\eta(s)))| d s \\
& +\left|\int_{\sigma(\tau)}^{\sigma(t)}\right| v(\tau, s)|h(s) d s| \\
& \leq|q(t)-q(\tau)|+\int_{0}^{\sigma(t)}|v(t, s)-v(\tau, s)| h(s) d s+|p(t)-p(\tau)|,
\end{aligned}
$$

where $p(t)=V \int_{0}^{\sigma(t)} h(s) d s$. Since $q$, $p$, and $k_{s}(t)=k(t, s)$ are continuous on $J$, they are uniformly continuous and consequently

$$
\left|B x_{n}(t)-B x_{n}(\tau)\right| \longrightarrow 0 \quad \text { as } t \longrightarrow \tau .
$$

Thus $\left\{B x_{n}: n \in \mathbb{N}\right\}$ is in equicontinuous set in $B M(J, \mathbb{R})$. Hence $B([u, v])$ is compact by Arzelá-Ascoli theorem for compactness. Thus $B$ is a continuous and compact operator on $[u, v]$. Finally, we have

$$
\begin{aligned}
M \phi_{A}(r)+\phi_{C}(r) & =\|B([u, v])\| \phi_{A}(r)+\phi_{C}(r) \\
& \leq\left[\left\|\alpha_{1}\right\|\left(Q+V\|h\|_{L^{1}}\right)+\left\|\beta_{1}\right\|\right] r<r
\end{aligned}
$$

for all $r>0$ because $\left\|\alpha_{1}\right\|\left(Q+V\|h\|_{L^{1}}\right)+\left\|\beta_{1}\right\|<1$.

Thus all the conditions of Theorem 2.3 are satisfied and hence an application of it yields that the operator equation (3.2) has a minimal solution and a maximal one in $[a, b]$. This further implies that the FIE (3.1) has a minimal solution and maximal one in $[a, b]$. This completes the proof.

\section{An application}

Consider the following initial value problem of first-order functional differential equation (in short FDE)

$$
\begin{aligned}
\left(\frac{x(t)-k(t, x(\mu(t)))}{f(t, x(\theta(t)))}\right)^{\prime} & =g(s, x(\eta(t))) \quad \text { a.e. } t \in J, \\
x(0) & =x_{0} \in \mathbb{R},
\end{aligned}
$$

where $f: J \times \mathbb{R} \rightarrow \mathbb{R} \backslash\{0\}$ is continuous, $f, k: J \times \mathbb{R} \rightarrow \mathbb{R}$, and $\mu, \theta, \eta: J \rightarrow J$ are continuous with $\theta(0)=0$.

By a solution of FDE (4.2)-(4.7), we mean a function $x \in A C(J, \mathbb{R})$ that satisfies equations (4.2)-(4.7), where $A C(J, \mathbb{R})$ is the space of all absolutely continuous real-valued functions on $J$.

We define an order relation $\leq$ in $A C(J, \mathbb{R})$ with the help of the cone $K_{A C}$ defined by

$$
K_{A C}=\{x \in A C(J, \mathbb{R}) \mid x(t) \geq 0 \forall t \in J\} .
$$

Clearly, the cone $K_{A C}$ is positive and normal in $A C(J, \mathbb{R})$. We need the following definition in the sequel. 
Definition 4.1. A function $u \in A C(J, \mathbb{R})$ is called a lower solution of FDE (4.2)-(4.7) if

$$
\begin{aligned}
\left(\frac{u(t)-k(t, u(\mu(t)))}{f(t, u(\theta(t)))}\right)^{\prime} & \leq g(s, u(\eta(t))) \quad \text { a.e. } t \in J, \\
u(0) & \leq x_{0} \in \mathbb{R} .
\end{aligned}
$$

Similarly, a function $v \in A C(J, \mathbb{R})$ is called an upper solution of (4.2)-(4.7) if

$$
\begin{gathered}
\left(\frac{v(t)-k(t, v(\mu(t)))}{f(t, v(\theta(t)))}\right)^{\prime} \geq g(s, v(\eta(t))) \quad \text { a.e. } t \in J, \\
v(0) \geq x_{0} \in \mathbb{R} .
\end{gathered}
$$

Theorem 4.2. Assume that the hypotheses $\left(\mathrm{H}_{3}\right)-\left(\mathrm{H}_{7}\right)$ hold. Further, suppose that

$$
\left\|\alpha_{1}\right\|\left(\left|\frac{x_{0}}{f\left(0, x_{0}\right)}\right|+\|h\|_{L^{1}}\right)+\left\|\beta_{1}\right\|<1
$$

Then FDE (4.2)-(4.7) has a minimal solution and a maximal one on J.

Proof. The FDE (4.2)-(4.7) is equivalent to the integral equation

$$
x(t)=k(t, x(\mu(t)))+[f(t, x(\theta(t)))]\left(\frac{x_{0}}{f\left(0, x_{0}\right)}+\int_{0}^{t} g(s, x(\eta(s))) d s\right), \quad t \in J .
$$

Now the desired conclusion follows by an application of Theorem 2.4 with $Q=$ $\left|x_{0} / f\left(0, x_{0}\right)\right|$ because $A C(J, \mathbb{R}) \subset B M(J, \mathbb{R})$. The proof is complete.

\section{References}

[1] H. Amann, Fixed point equations and nonlinear eigenvalue problems in ordered Banach spaces, SIAM Rev. 18 (1976), no. 4, 620-709.

[2] D. W. Boyd and J. S. W. Wong, On nonlinear contractions, Proc. Amer. Math. Soc. 20 (1969), 458-464.

[3] S. Chandrasekhar, Radiative Transfer, Dover Publications, New York, 1960.

[4] K. Deimling, Nonlinear Functional Analysis, Springer-Verlag, Berlin, 1985.

[5] B. C. Dhage, Some nonlinear alternatives in Banach algebras with applications I, to appear in Nonlinear Stud.

[6] Some fixed point theorems in ordered Banach spaces and applications, Math. Student 61 (1992), no. 1-4, 81-88.

[7] _ Fixed point theorems in ordered Banach algebras and applications, Panamer. Math. J. 9 (1999), no. 4, 83-102.

[8]_, On existence theorems for nonlinear integral equations in Banach algebras via fixed point techniques, EAMJ East Asian Math. J. 17 (2001), no. 1, 33-45.

[9] B. C. Dhage and D. O'Regan, A fixed point theorem in Banach algebras with applications to functional integral equations, Funct. Differ. Equ. 7 (2000), no. 3-4, 259-267.

[10] A. Granas, R. B. Guenther, and J. W. Lee, Some general existence principles in the Carathéodory theory of nonlinear differential systems, J. Math. Pures Appl. (9) 70 (1991), no. 2, 153-196.

[11] D. J. Guo and V. Lakshmikantham, Nonlinear Problems in Abstract Cones, Notes and Reports in Mathematics in Science and Engineering, vol. 5, Academic Press, Massachusetts, 1988. 


\section{Extremal solutions of integral equations}

[12] S. Heikkilä and V. Lakshmikantham, Monotone Iterative Techniques for Discontinuous Nonlinear Differential Equations, Monographs and Textbooks in Pure and Applied Mathematics, vol. 181, Marcel Dekker, New York, 1994.

[13] M. A. Krasnosel'skii, Topological Methods in the Theory of Nonlinear Integral Equations, Pergamon Press Book, The Macmillan, New York, 1964.

B. C. Dhage: Kasubai, Gurukul Colony, Ahmedpur 413 515, Maharashtra, India

E-mail address: bcd20012001@yahoo.co.in 


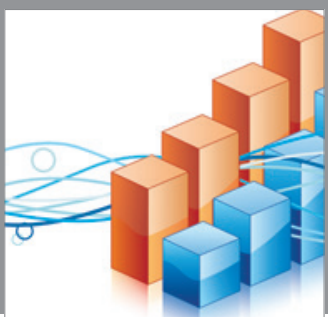

Advances in

Operations Research

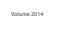

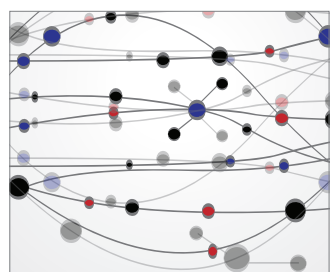

\section{The Scientific} World Journal
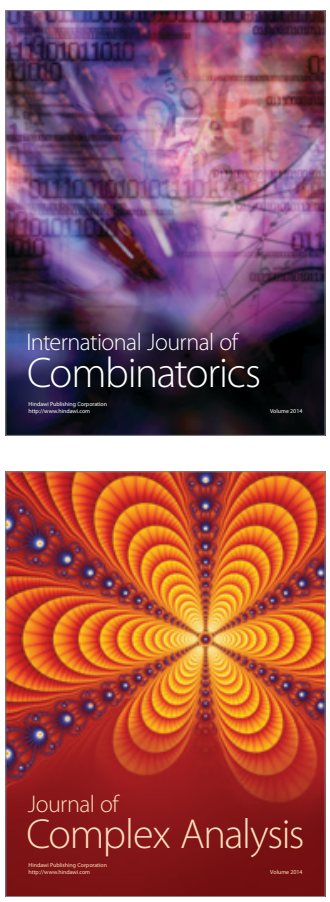

International Journal of

Mathematics and

Mathematical

Sciences
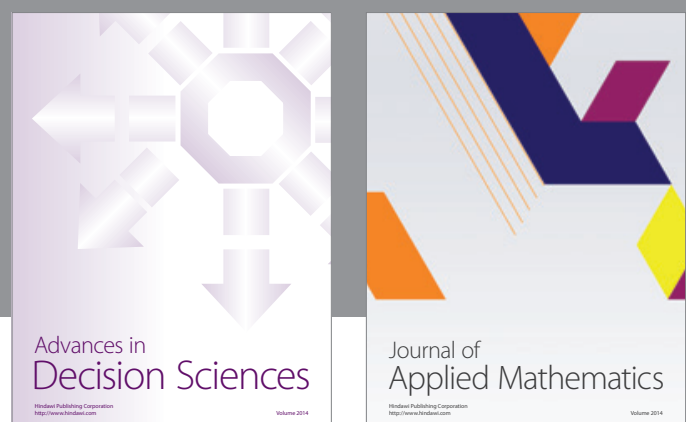

Journal of

Applied Mathematics
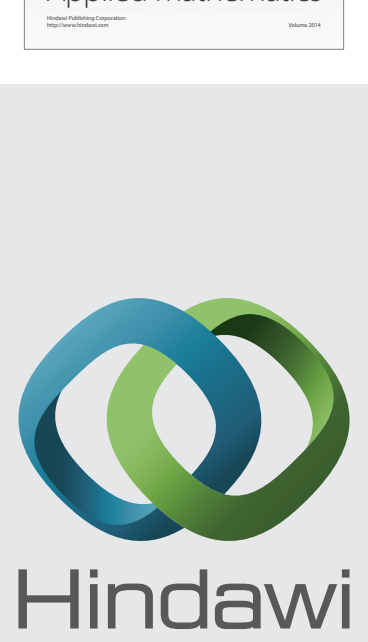

Submit your manuscripts at http://www.hindawi.com
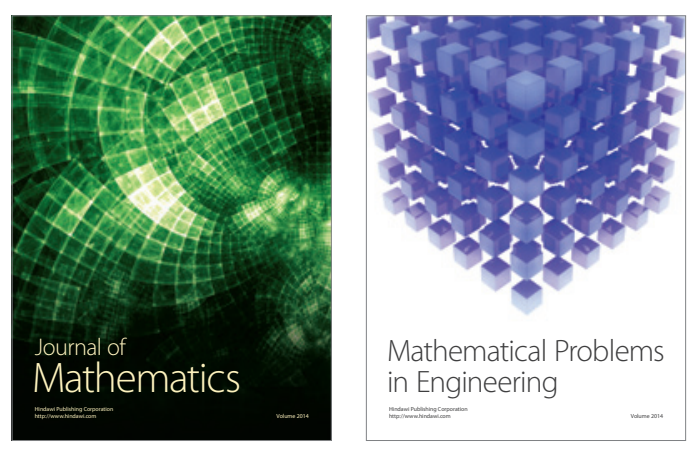

Mathematical Problems in Engineering
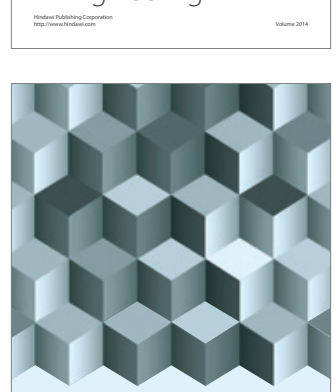

Journal of

Function Spaces
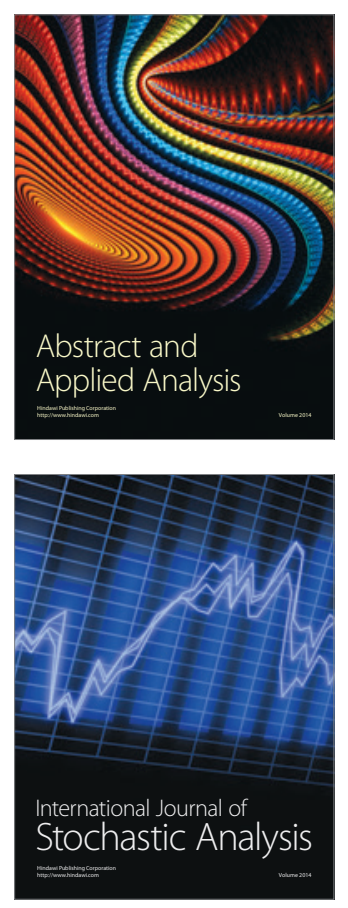

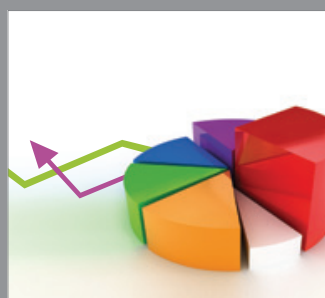

ournal of

Probability and Statistics

Promensencen
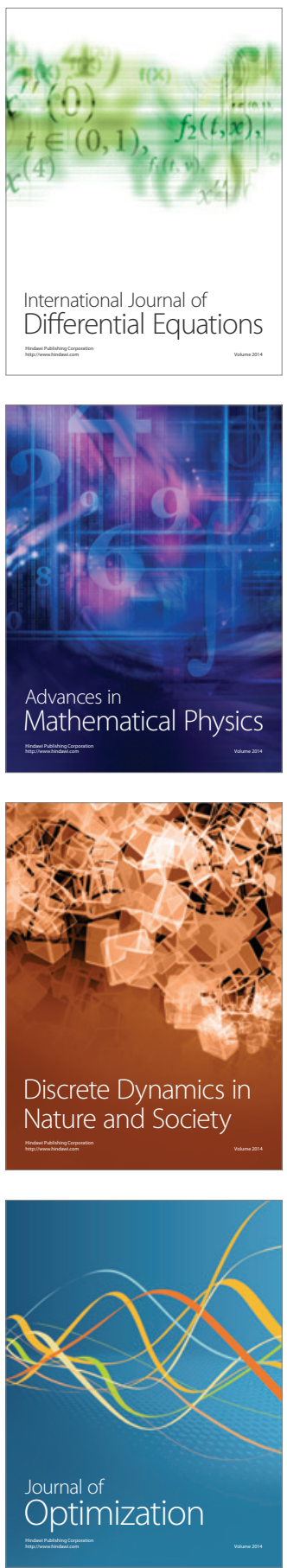